\title{
A portable secondary dose monitoring system using scintillating fibers for proton therapy of prostate cancer: A Geant4 Monte Carlo simulation study
}

\author{
Biniam Tesfamicael' ${ }^{1}$ Paul Gueye ${ }^{2}$, Stephen Avery ${ }^{3}$, Donald Lyons ${ }^{3}$, Mahadevappa Mahesh ${ }^{4}$ \\ ${ }^{1}$ Department of Radiation Oncology, McLaren Regional Medical Center, Flint, Michigan, USA \\ 2 Department of Physics, Hampton University, Hampton, Virginia, USA \\ ${ }^{3}$ Department of Radiation Oncology, University of Pennsylvania, Philadelphia, Pennsylvania, USA \\ ${ }^{4}$ Department of Radiology and Radiological Sciences, John Hopkins University, Baltimore, Maryland, USA
}

Received July 05, 2015; Revised January 29, 2016; Accepted February 07, 2016; Published Online February 12, 2016

\section{Original Article}

\begin{abstract}
Purpose: The main purpose of this study was to monitor the secondary dose distribution originating from a water phantom during proton therapy of prostate cancer using scintillating fibers. Methods: The Geant4 Monte Carlo toolkit version 9.6.p02 was used to simulate a proton therapy of prostate cancer. Two cases were studied. In the first case, $8 \times 8=64$ equally spaced fibers inside three $4 \times 4 \times 2.54$ $\mathrm{cm}^{3}$ Delrin $\AA$ blocks were used to monitor the emission of secondary particles in the transverse (left and right) and distal regions relative to the beam direction. In the second case, a scintillating block with a thickness of $2.54 \mathrm{~cm}$ and equal vertical and longitudinal dimensions as the water phantom was used. Geometrical cuts were implemented to extract the energy deposited in each fiber and inside the scintillating block. Results: The transverse dose distributions from the detected secondary particles in both cases are symmetric and agree to within $<3.6 \%$. The energy deposited gradually increases as one moves from the peripheral row of fibers towards the center of the block (aligned with the center of the prostate) by a factor of approximately 5 . The energy deposited was also observed to decrease as one goes from the frontal to distal region of the block. The ratio of the energy deposited in the prostate to the energy deposited in the middle two rows of fibers showed a linear relationship with a slope of $(-3.55 \pm 2.26) \times 10^{-5} \mathrm{MeV}$ per treatment Gy delivered. The distal detectors recorded a negligible amount of energy deposited due to higher attenuation of the secondary particles by the water in that direction. Conclusion: With a good calibration and with the ability to define a good correlation between the radiation flux recorded by the external fibers and the dose delivered to the prostate, such fibers can be used for real time dose verification to the target. The system was also observed to respond to the series of Bragg Peaks used to generate the Spread Out Bragg Peak inside the water phantom. Such Bragg Peaks were detected by the fibers. The energy deposited inside the lateral blocks were also observed to decrease as one goes away from the beam nozzle due to increased attenuation.
\end{abstract}

Keywords: Proton Therapy; Prostate Cancer; Scintillating Fibers; Geant4; Hadrontherapy; Secondary Dose

\section{Introduction}

Radiation therapy has been one of the most commonly used treatment options for cancer patients. The external beam radiation therapy is performed using high energy photons as well as charged particles, mainly electrons and protons. Due to the sharp characteristic Bragg peak it possesses and the technological ability to spread the 
Bragg Peak to cover the entire tumor size in the beam direction, proton therapy has shown a therapeutic advantage over the conventional photon therapy in sparing much of the surrounding healthy tissue in the treatment of deep seated malignancies like the prostate cancer. $^{\mathbf{1 - 8}^{-8}}$ In proton therapy, the beam can be delivered in either an active mode or passive mode. ${ }^{5-7}$ In the former, an aperture and a compensator that are generated from the treatment planning system are fabricated and utilized to conform the beam to the target shape both laterally and in the beam direction, respectively.

The major concern regarding proton therapy, however, has been the production of secondary particles, especially neutrons, through inelastic interactions of the energetic protons with the beam delivery system components and the patient body.9,10 The former was found to be the major source of neutrons.9,10 The dose delivered to patients by such secondary particles has been of great concern and a main topic of study by a number of researchers., ${ }^{41-15}$ Results showed that the dose from neutrons is very low and the secondary cancer incidence from such a dose is very scarce. ${ }^{16}$ The production of neutrons was found to be high when using a passive beam delivery mode. This is due to presence of the scatterers and apertures, which are the main contributors in neutron production in the beam line. Binns et al.12 measured the neutron dose equivalent at the patient position to be in the range of $33 \mathrm{mSv}$ to 80 mSv per treatment Gy when a proton beam of $200 \mathrm{MeV}$ mean energy was delivered using a passive beam delivery mode. Around the treatment nozzle, an equivalent dose of $0.91 \mathrm{mSv}$ to $15 \mathrm{mSv}$ per treatment Gy was measured at the Harvard Cyclotron Laboratory by Yan et al. ${ }^{14}$ from $160 \mathrm{MeV}$ proton beam with a passive beam delivery mode.

The equivalent dose from secondary neutrons decreases with a decrease in the treatment field, an increase in the distance from the nozzle as well as a decrease in the energy of the primary proton beam. Agosteo et al.11 reported a Monte Carlo simulation estimation of a maximum neutron dose of $10^{-4} \mathrm{~Gy}$ per treatment Gy in the healthy tissue behind the eye from a passively scattered ocular treatment beam line using a $65 \mathrm{MeV}$ proton beam at the Center Antoine-Lacassagne in France. With an active beam delivery mode, the absence of scatterers and apertures greatly reduces the neutron production. ${ }^{17}$ Uwe et al. ${ }^{15}$ measured the dose delivered to the healthy tissue by the secondary neutrons for large and medium targets to be approximately $4 \mathrm{mSv}$ and 2 $\mathrm{mSv}$ per treatment Gy, respectively when a $177 \mathrm{MeV}$ proton beam was used to irradiate the tumor in a spot scanning beam delivery mode.

In this work, a Geant4 Monte Carlo simulation toolkit ${ }^{18,19}$ was used to study the distribution of the secondary particles around a $36 \mathrm{~cm} \times 22 \mathrm{~cm} \times 24 \mathrm{~cm}$ water phantom. The energy deposited around the water phantom by these secondary particles was analyzed. The dose monitoring system constructed in this study is mainly based on the energy deposited external to the water phantom. This work follows a recent paper that focused in the dose distribution near and in the prostate gland.$^{20}$ In the previous paper, a dose monitoring system was constructed to study the dose delivered to the rectal wall in the proton therapy of prostate cancer. The simulation included thin scintillating fibers attached to an endorectal balloon to record the dose delivered to the balloon surface. This represents the dose to the inner rectal wall, which is in physical contact with the balloon. The results obtained indicated that a good correlation can be built between the dose delivered to the prostate and the dose to the scintillating fibers, thus an in-vivo dose monitoring to the rectal wall as well as the target prostate can be achieved.

\section{Methods and Materials}

The Geant4 Monte Carlo toolkit version 4.9.6 p02 was used to simulate a proton therapy of prostate cancer with an endorectal balloon as an internal immobilizer. The simulation was based on the hadrontherapy example application available with the Geant4 package ${ }^{21-25}$, modified for the present study. The details of the simulation developed are described in the previous paper. ${ }^{20}$ For the secondary dose monitoring system, three detectors were constructed around the water phantom. From the beam's eye view, two detectors were placed in the lateral directions and one in the distal. Two sets of detectors were designed for this study. In the first case, a $4 \mathrm{~cm} \times 4 \mathrm{~cm}$ Delrin $\AA$ block was used to house a total of $8 \times 8=64$ fibers of dimension $1 \mathrm{~mm} \times 1 \mathrm{~mm}$, evenly spaced by a center-tocenter distance of $4.556 \mathrm{~mm}$ from each other both in the vertical and lateral directions. The length of the fibers was defined by the thickness of the Delrin $®$ block, which is $2.54 \mathrm{~cm}$. In the second case, a $36 \mathrm{~cm} \times 22 \mathrm{~cm} \times 2.54 \mathrm{~cm}$ block made entirely of scintillating material was used for radiation detection. Each block was aligned to the center of the prostate. In both cases, the detectors were placed at a distance of $4 \mathrm{~cm}$ away from the surface of the water phantom. The placement of the scintillating fibers inside the Delrin $\AA$ block and the scintillating blocks are shown in Figures 1 and 2.

The modulator wheel was rotated from $0^{0}$ to $359^{0}$ in steps of $1^{0}$ to spread out the Bragg Peak in the beam direction. The beam was laterally conformed to the target shape using patient specific collimator located at the end of the beam line. With the Delrin $®$ block, the simulation was run for four different number of events per degree of the modulator wheel rotation: 500, 1000, 2000,3000 . The results obtained were analyzed to study the linear response of the scintillating detectors to the dose delivered. For the case of the scintillating block, however, the simulation was run solely for 3000 events 
per degree of modulator wheel rotation to evaluate the effect of Delrin $®$ material as a host to the dose monitoring fibers as in the former case.

\section{Results}

The fibers were uniformly spaced within the blocks, with the fiber ID running from 1 to 64 as shown in Figure 1. The beam direction is from left to right, irradiating the whole prostate. In the root file ${ }^{26}$ generated from the simulation run, the energy deposited in all the fibers inside the three blocks were returned under one tree by implementing a cut in the volume name. To analyze the energy deposited in each fiber, the knowledge of the geometrical location of the individual fibers inside the blocks is required. The plots of the interaction points in the fibers in the $X-Y$ (along the beam direction) and Z-Y (along the length of the fibers) planes in the lateral blocks are shown in Figure 2. The geometry simulating the prostate is centered at $\mathrm{Y}=\mathrm{Z}=0$ and $X=21.5 \mathrm{~cm}$. The right block $(Z>0)$ is the reflection of the left block $(\mathrm{Z}<0)$ with the $\mathrm{X}$-axis as a mirror at $\mathrm{Z}=$ 0 . Hence, it is sufficient to obtain the information regarding the geometric location of the fibers from one block only. The information for the other lateral block fibers will be a mirror reflection of the first block fibers along the $\mathrm{X}$-axis, i.e. setting $\mathrm{Z}>0$ will give the coordinates of the fibers in the right block and $\mathrm{Z}<0$ will be that of the fibers in the left block from the beam's eye view.

From the two plots above, the coordinates that define the volume of the individual fibers was generated. The information was later used to calculate the total integrated energy deposited in each fiber located inside the left and right blocks.

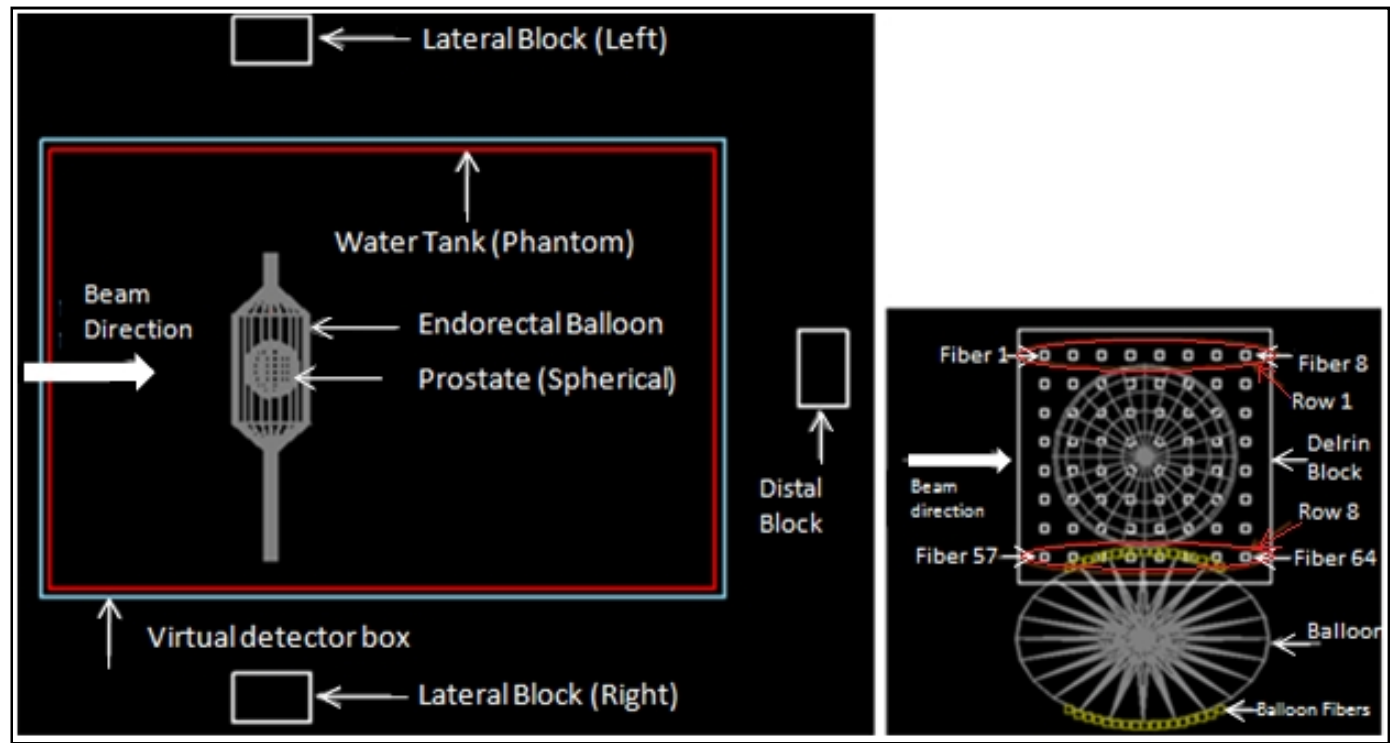

Figure 1: Left panel: the top view of the simulation design in and around the water phantom. Right panel: the placement of the 64 scintillating fibers inside the lateral Delrin ${ }^{\circledR}$ blocks. The blocks are aligned to the center of the prostate and were placed $4 \mathrm{~cm}$ away from the surface of the water phantom both in the two lateral and distal regions.
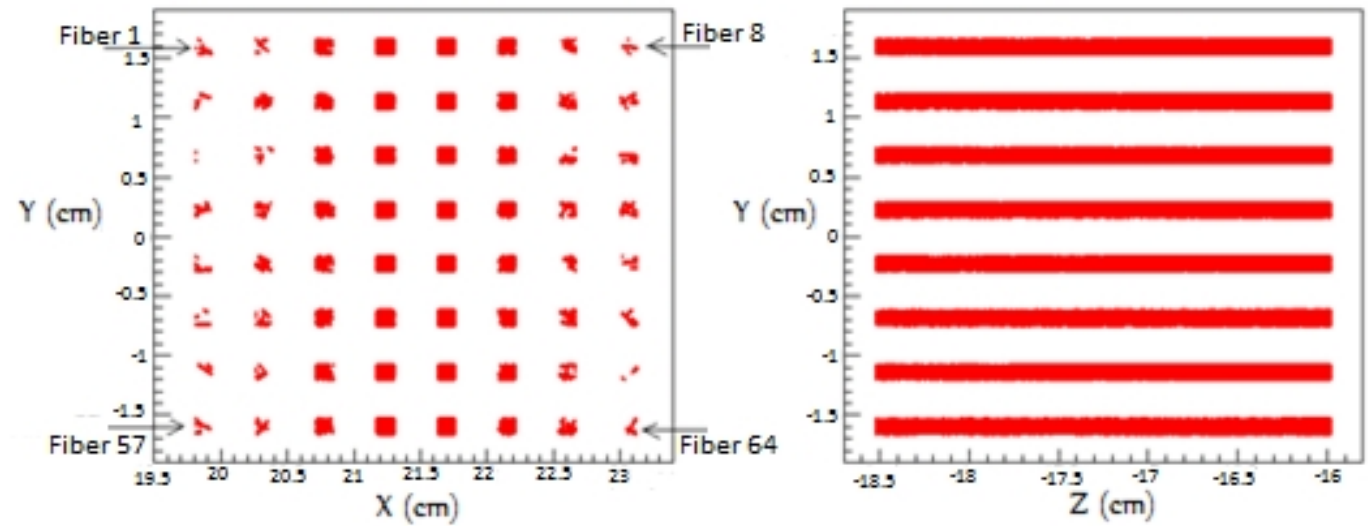

Figure 2: The interaction points inside the left block fibers along the fiber length (Y-Z plot) and in (Y-X) the beam direction (beam direction is along the $\mathrm{X}$ axis). 


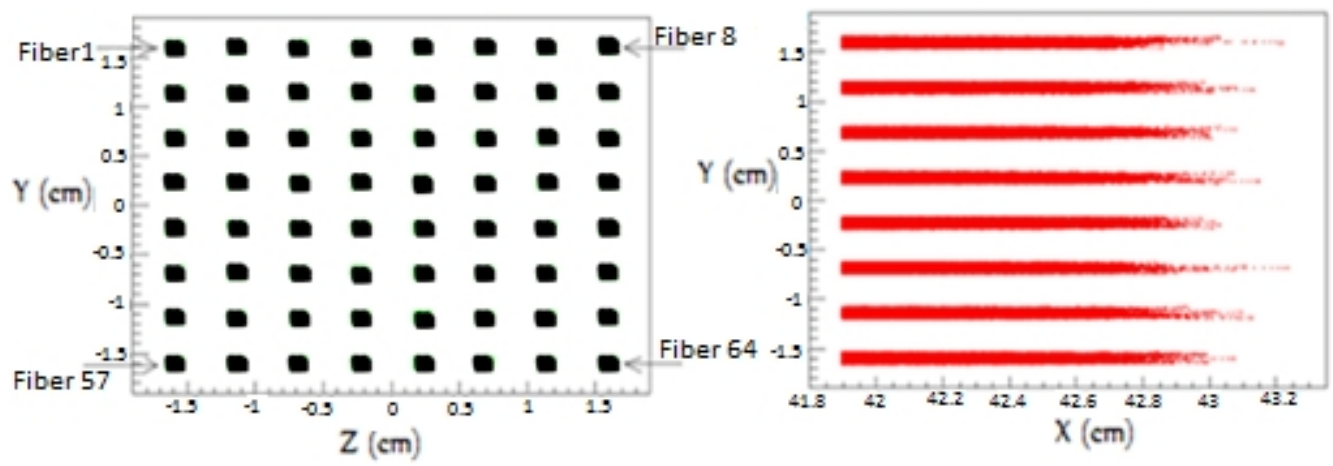

Figure 3: The interaction points of the radiation within the distal block fibers. The fiber ID goes from 1 to 64 from the top-left to the bottom-right corner.

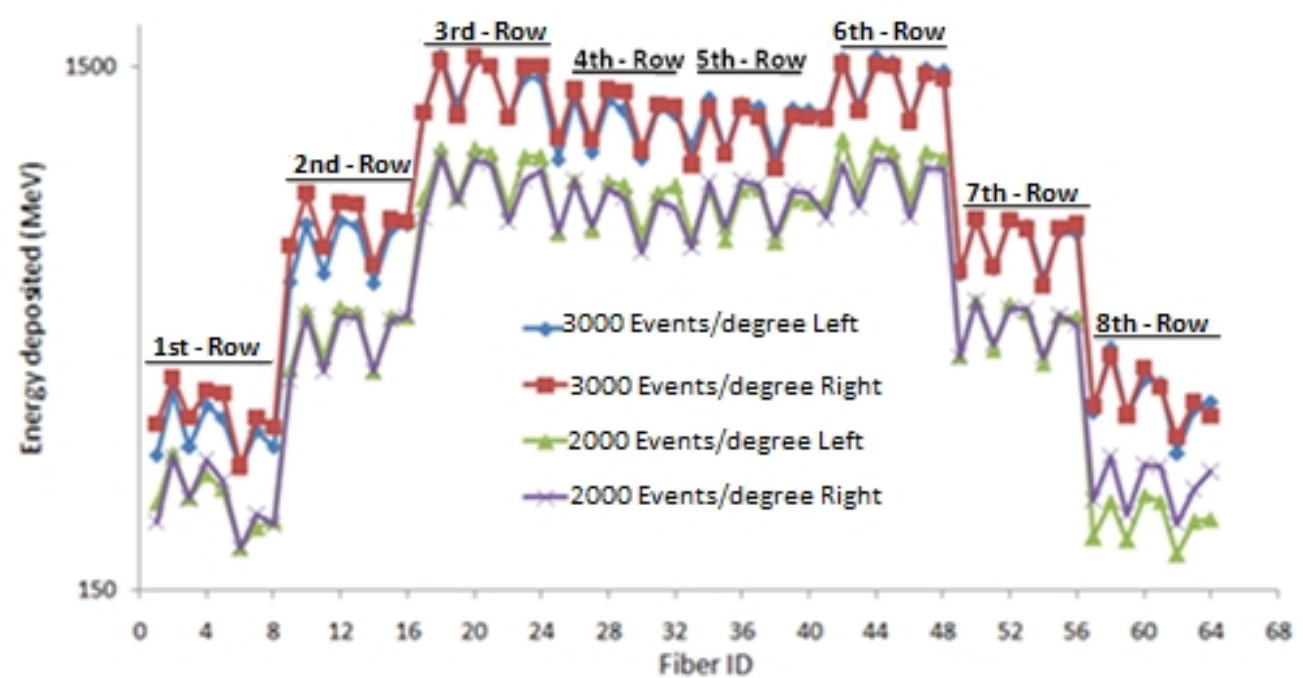

Figure 4: The total integrated energy deposited inside the right and left fiber within the Delrin® block for the 2000 and 3000 events per degree of modulator rotation. The same results were observed for the 500 and 1000 events per modulator wheel rotation - (not shown for clarity).

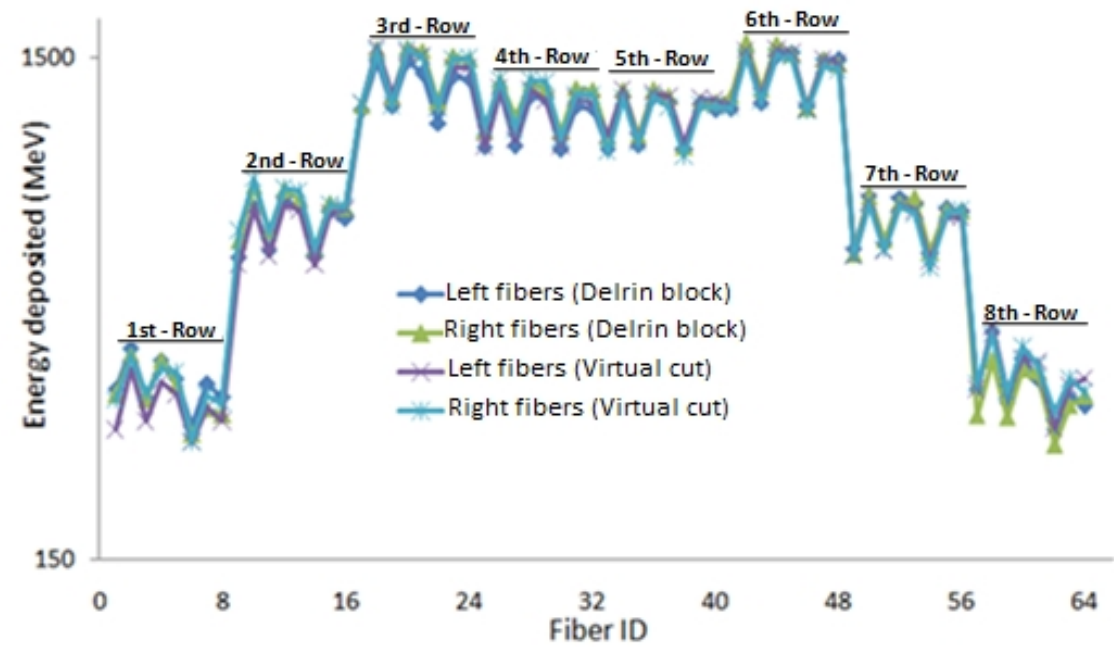

Figure 5: The energy deposited in the left and right fiber blocks with and without the virtual cut. Both simulation runs were for 3000 events per degree of modulator rotation. 


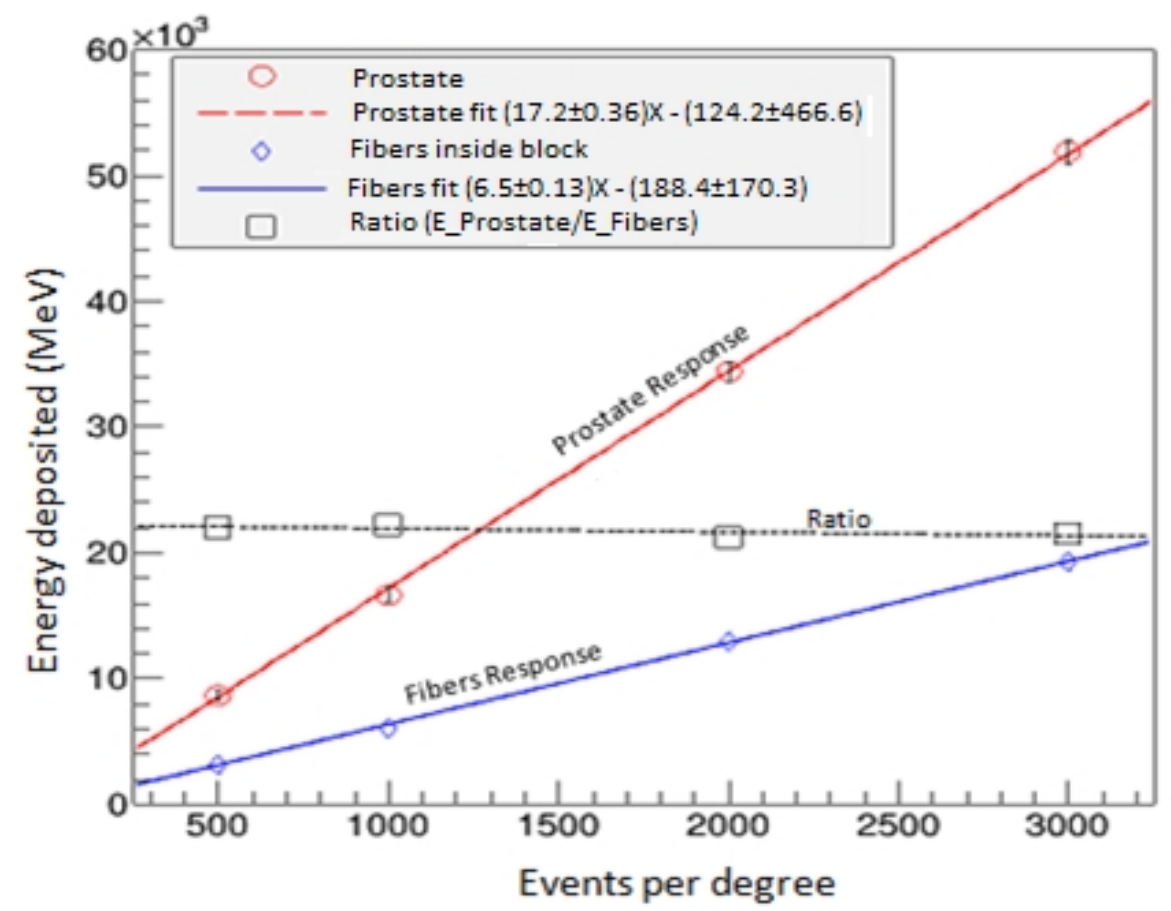

Figure 6: The energy deposited in the prostate and the total energy in the 16 fibers located in rows 4 and 5 above ( $+1.8 \mathrm{~mm})$ and below $(-1.8 \mathrm{~mm})$ the center of the prostate, respectively. The plot also shows the ratio of the two energies deposited (E_Prostate/E_fibers), which is scaled up by 8,000 for clarity.

For the fibers in the distal region, the beam was moved forward with its momentum in the positive $\mathrm{X}$ direction. Such setup results in more interaction points inside the distal block fibers, hence giving a clear image of the geometry of the fibers. From the plot of the interaction points, the geometrical information regarding the 64 fibers was generated and later used to calculate the total integrated energy deposited inside each fiber. The plot of the interaction points in the fibers inside the distal block are shown in Figure 3.

Once the geometrical coordinates for all the fibers were extracted, the simulation was run for the different number of events per modulator wheel rotation for proton beam energy of $200 \mathrm{MeV}$ as listed in section II. The plot of the integrated energy deposited in the fibers for the lateral blocks is shown in Figure 4.

Figure 4 shows a clear symmetry in the energy deposited between the left and right block fibers. The plot also shows symmetry in the energy deposited in the fibers across the rows. The fibers in the first row, which are physically located at the top of the block (see Figure 1 ), record relatively the same amount of energy deposited as those in the eighth row, which are located at the bottom of the block. The same relationship exists between the second and seventh, third and sixth as well as fourth and fifth row fibers. Such pairs of row fibers are located at the same distance from the center of the prostate.
The energy deposited in the fibers increase from row 1 (and 8) to row 3 (and 6), then slightly decreases in row 4 (and 5). Fibers in rows 1 and 8 are at comparatively longer distances from the center of the prostate. Hence, the secondaries will travel a longer distance to arrive at those fibers, experiencing a relatively higher attenuation. For the fibers in rows 3 and 6, the distance decreases, resulting in lower attenuation and higher deposited energy as compared to the energy recorded by the fibers in rows 1 and 8 . The energy deposited continues to increase as one move from the edge, rows 1 and 8 , towards the middle, rows 4 and 5. A slight decrease in the energy deposited in rows 4 and 5 could be attributed to the spherical geometry of the prostate, which makes it thicker at the center. This affects the energy deposited inside the fibers in rows 4 and 5 that are located slightly above and below the center of the prostate, respectively.

In the second case, the Delrin $₫$ block was replaced by a full solid scintillating slab of dimensions $36 \mathrm{~cm} \times 22 \mathrm{~cm}$ $\times 2.5 \mathrm{~cm}$. This simulation was run for 3000 events per modulator rotation and virtual cuts were applied to obtain the energy deposited in the geometrical location of the fibers. The geometrical information of the fibers generated from Figure 2 was used for the virtual cut and the integrated energy deposited was calculated and is depicted in Figure 5.

The plots overlap very well indicating that the Delrin ${ }^{\circledR}$ material used to house the individual fibers has no effect 
on the reading of the fibers. In the four different runs, the energy deposited in the prostate as well as the total energy deposited in the sixteen fibers in rows 4 and 5 were recorded and analyzed. The plot of the energy deposited as a function of the number of events per modulator wheel rotation (i.e., dose) for both the prostate and these fibers is shown in Figure 6. Linear fits applied for each plot showed a slop of $17.2 \pm 0.35$ for the prostate and $6.5 \pm 0.13$ for the fibers in the two middle rows.
The plot for the ratio of the energy deposited inside the prostate to that recorded by the fibers is relatively constant, with a slope of $(-3.55 \pm 2.26) \times 10^{-5} \mathrm{MeV}$ per treatment Gy to the prostate. The results obtained ensures a direct scaling between the two energies deposited. Thus, defining a precise correlation between the energy deposited in the prostate and the energy deposited in the 16 fibers located outside of the water phantom will enable one to get a good prediction of the dose to the prostate from the measurement of the radiation flux in the fibers.

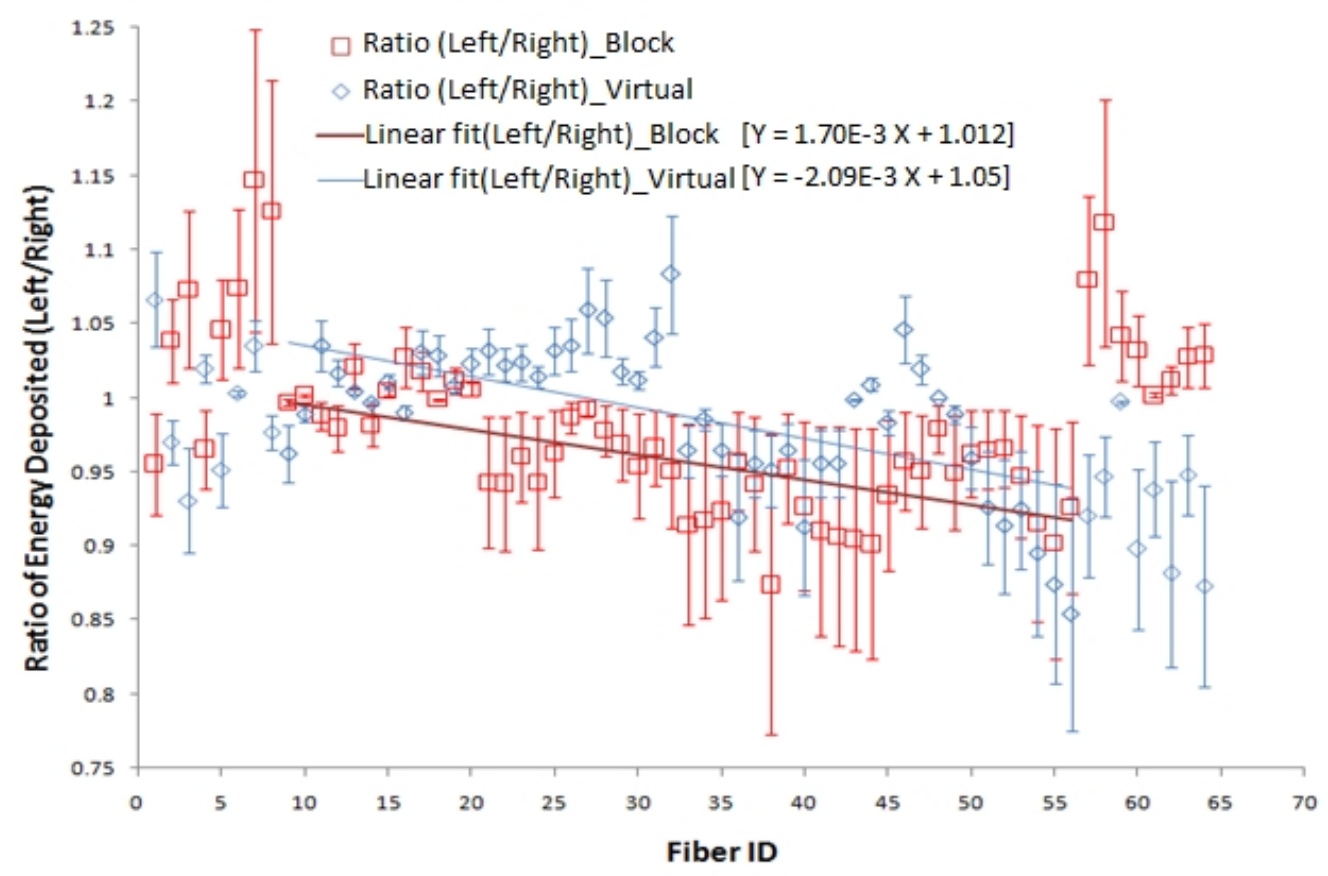

Figure 7: The ratio of the energy deposited for the 3000 events per degree of modulator wheel rotation run. The plot shows the ratio of the energy deposited in the left fibers to those in the right fibers for both the block and virtual cut cases.
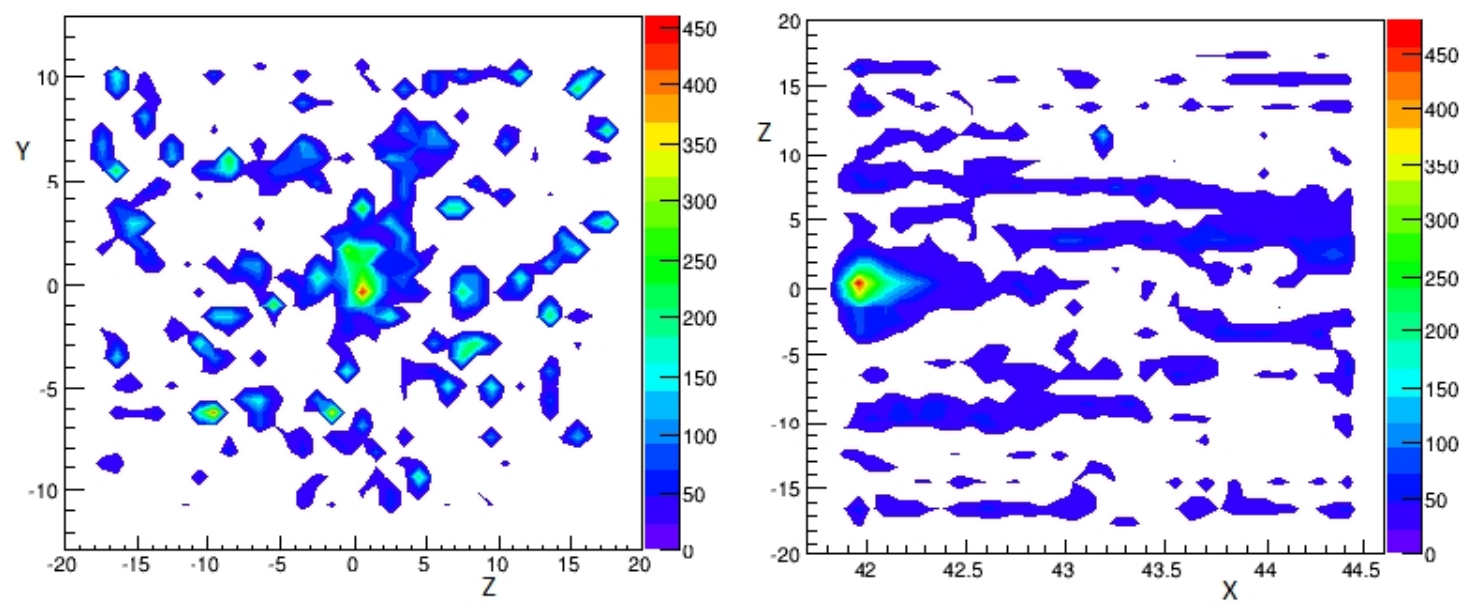

Figure 8: Contour plot of the interaction points in the distal scintillating block. Beam direction in the X Axis. Left panel is in beam's eye view and Right panel transverse to beam direction. 


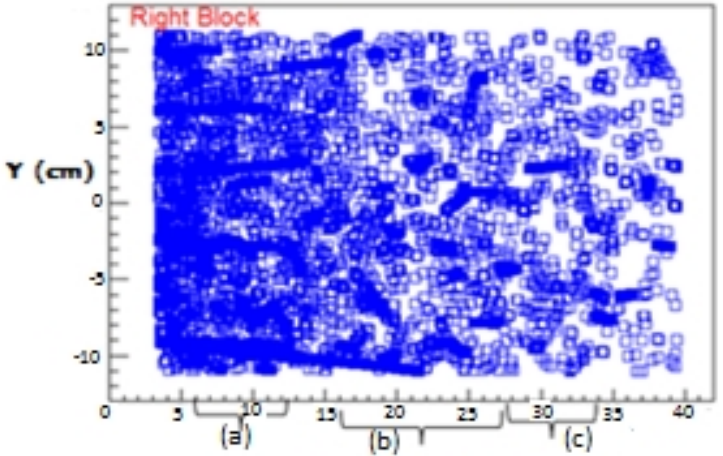

Position along beam Axis $(\mathrm{cm})$

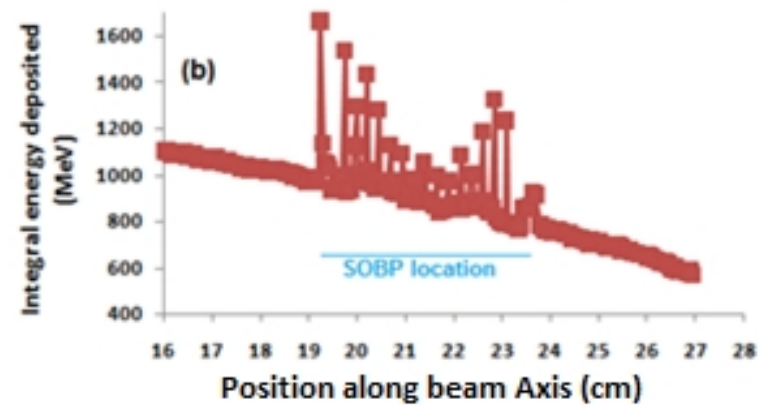

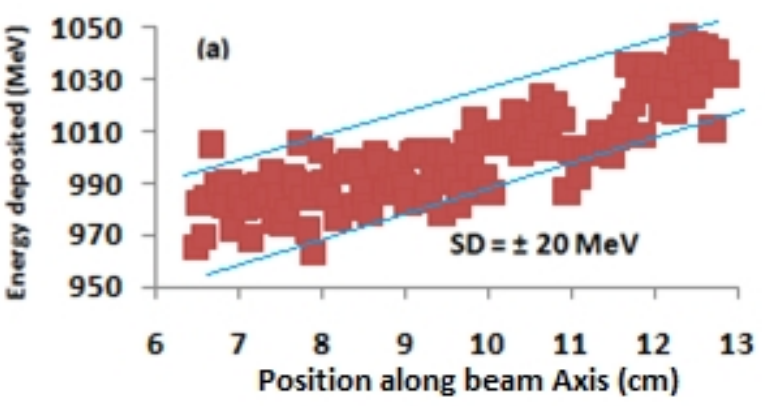

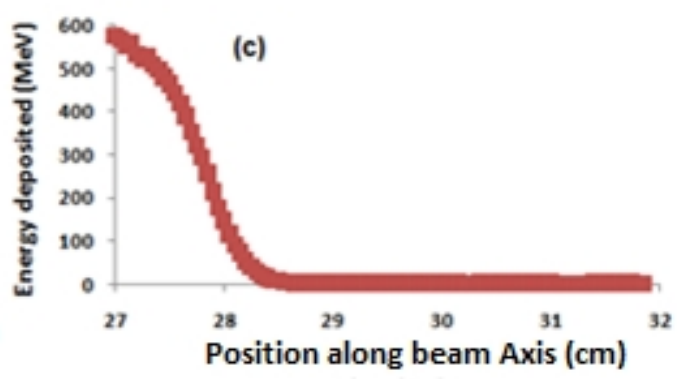

Figure 9: The energy deposited in thin slabs of the right scintillating block at different locations along the beam direction.

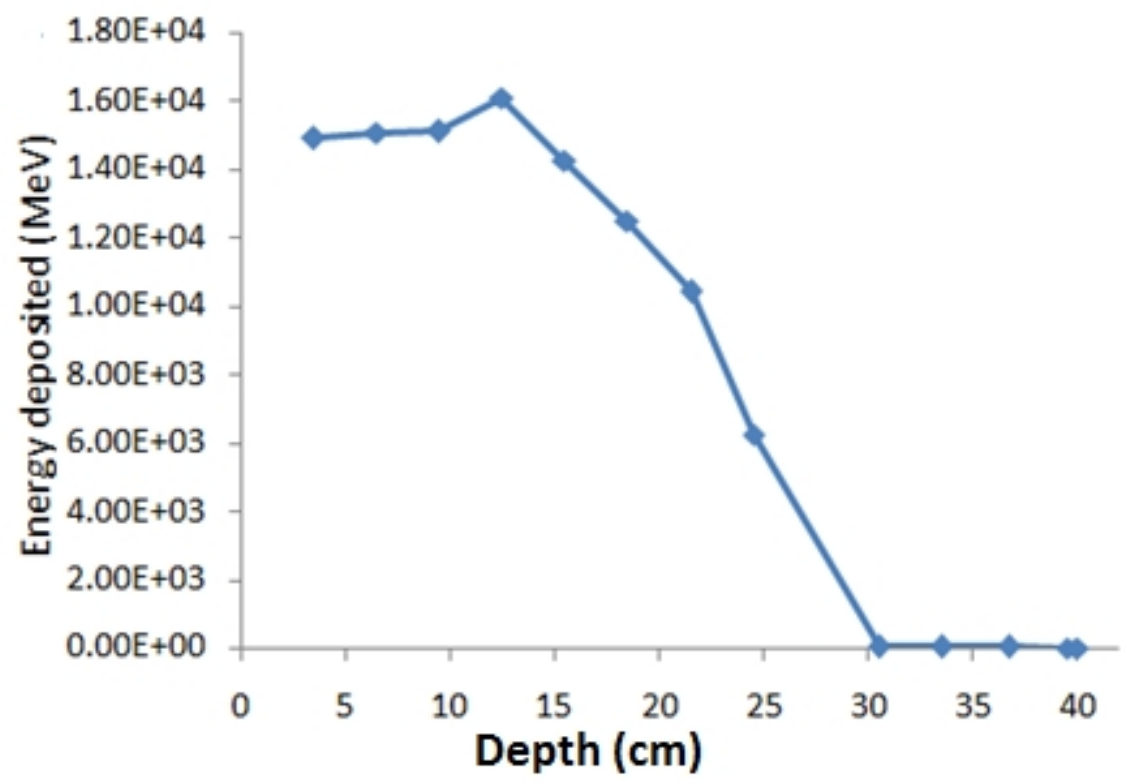

Figure 10: The energy deposited as a function of depth in the lateral region.

\section{Discussion}

From the results obtained, one could notice a definite symmetry in the distribution of the secondary particles in the lateral (right and left) regions in both cases as depicted in Figures 4 and 5. This indicates a uniform distribution of the radiation exiting the water phantom. The plot of the ratio of the energy deposited in the left and right regions for both cases is shown in Figure 7.
Figure 7 shows the ratio of the energy deposited in the left to that of the right fibers for both cases (inside the Delrin ${ }^{\circledR}$ block and for the full scintillating slab). The data analysis showed that the flux in the two lateral regions match to within $3.6 \%$ in both cases. The linear fit performed over a selected region also showed a very small slope, with a y-intercept close to 1 , with the two

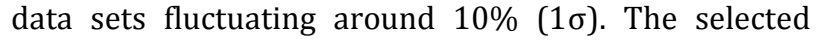


region for the linear fit was the fibers located between rows 2 and 7, where there is sufficient statistics.

Moreover, the ratio of the data obtained from the fibers inside the Delrin ${ }^{\circledR}$ block to that of the virtual cuts inside the scintillating slab was analyzed for both regions. The data match to within $3.5 \%$, which indicates that the material housing the dose monitoring fibers has less effect on the energy deposited inside those fibers.

The secondary dose monitoring fibers and the scintillating block in the distal region, however, recorded very minimum energy deposited. This is due to the higher attenuation the secondary particles experience in arriving at those fibers for the selected beam energy. The primary purpose of this detector was to obtain information on the beams centroid in order to provide a correction on the beam alignment.

To generate the contour plot in Figure 8, a proton beam of energy $250 \mathrm{MeV}$ was used with the beam source positioned at around the center of the water phantom to overcome the higher attenuation. Observed from the beam's eye view, the left panel shows the detection of more secondaries around the center. The right panel shows the side view of the scintillating block detector, i.e. transverse to the beam direction. More secondaries were detected at the frontal edge around the center of the detector.

Further analysis of the data showed that the energy deposited in the fibers located in each row fluctuates, following a relatively similar pattern in all the rows. As shown in Figures 4 and 5, the "up-down" pattern appears in both cases, i.e. when using a Delrin $\AA$ block to house the monitoring fibers as well as when the whole scintillating block was used with a virtual cut. The fibers that are housed inside the Delrin ${ }^{\circledR}$ block in each row were spaced at $4.6 \mathrm{~mm}$ from each other. To understand this fluctuation, the scintillating block located on the right side of the water phantom, viewed from the beam's eye view, was virtually dissected in the vertical direction (slices run along beam direction) in thicknesses of 0.5 $\mathrm{mm}$. In the $\mathrm{Y}$ and $\mathrm{Z}$ directions (height and thickness, respectively), the whole size of the block was considered. Such slices $(0.5 \mathrm{~mm} \times 22 \mathrm{~cm} \times 2.45 \mathrm{~cm})$ were used to calculate the integrated energy deposited and the results obtained are shown in Figure 9.

The panel in the top left shows the scattered plot of the secondaries within the scintillating block. Panel (a) represents the energy distribution in the proximal region. The spread in the energy variation is equivalent to one standard deviation (SD). Hence, this variation is attributed to statistical fluctuation. In (b), however, the fluctuation follows a certain pattern that repeats over a given region, i.e. around the location of the spread out Bragg peak (SOBP). The repetitive spikes correspond to the large number of Pristine Bragg peaks involved to generate the SOBP (18 in number). The SOBP was generated by a modulator wheel of 18 slices, each with a thickness of $0.3 \mathrm{~cm}$ stacked together to form a staircase. The secondary dose monitoring fibers as well as the scintillating block located outside of the water phantom were able to "sense" those Pristine Bragg Peaks, resulting in the formation of the spikes. In (c), which is located in the distal region of the right block, no such spikes were observed due to attenuation and smearing in water. Such spike patterns were also observed inside the prostate. The spikes generated, however, were less pronounced due to a higher contribution of low energy secondaries that are suppressed from reaching the external detectors due to attenuation.

From Figures 9(a,b and c), one notices that the energy deposited gradually decreases as one moves towards the distal section of the scintillating block. To understand the distribution of the energy deposited, further analysis of the data was conducted. In this case, the scintillating block was virtually dissected into thin slices of thickness $3.5 \mathrm{~cm}$ each along the beam direction. The energy deposited in each slice was calculated and plotted as shown in Figure 10.

From the plot, it can be noticed that the energy deposited is higher in the proximal region and decreases towards the distal region. The peak at the depth of around $12 \mathrm{~cm}$ shows a turning point, which is in agreement to the plots in Figure 9 (a) and (b). Beyond that point, the energy deposited starts decreasing and is close to zero at depths of around $30 \mathrm{~cm}$ and beyond. This is due to the attenuation of the mainly low energy secondaries that are generated at depths beyond the location of the SOBP. The result obtained is in close agreement with those obtained by Wroe et al. ${ }^{27}$, who used a different setup for their experiment. The reason attributed for higher energy deposited in the proximal region is due to the higher production of high energy secondaries, like neutrons and electrons inside the beam line components which are closer to the proximal edge of the scintillating block. For the distal region, however, the main contribution comes from the secondary particles generated inside the water phantom. The secondaries from the water phantom are produced with comparatively lower energies.

\section{Conclusion}

A Geant4 Monte Carlo simulation was designed to monitor the total external radiation flux and a possible correlation of the measured flux with the dose delivered to the prostate. The previous work $^{20}$ was mainly focusing on using thin scintillating fibers attached to a water filled endorectal balloon to monitor the dose to the rectal wall as well as the prostate. In this work, however, the goal was to develop a dose monitoring system based on the radiation flux recorded from outside of the water phantom. 
The results obtained were in good agreement to results from literature review. A linear response of the fibers to the dose delivered to the prostate was observed, a property of the fibers studied by a number of researchers. Secondary dose monitoring with scintillating fibers could be used to give a relatively good prediction (with a precision higher than 97\%) of the dose to the prostate. The multiple Bragg peaks used during clinical treatment to generate an SOBP could be observed and monitored to estimate the non-uniform distribution of the dose within the SOBP region. Further studies on the biological effect of such dose none uniformity distribution is planned.

The grant allocated for this study was mainly for prostate cancer case. The simulation, however, can be modified, with little work, to study other cases. In the future, there is a plan by the authors to apply the Monte Carlo simulation developed to investigate the treatment of other anatomical sites. The authors are also looking for a fund for equipment purchase and beam time to conduct experimental measurements based on the simulation design. The results from the experimental measurements will be compared with the results from the simulation.

\section{Conflict of interest}

The authors declare that they have no conflicts of interest. The authors alone are responsible for the content and writing of the paper.

\section{Acknowledgement}

This work was partly supported by the Department of Energy - National Security Administration under award number DE-NA0000979.

\section{References}

1. Wilson RR. Radiological use of fast protons. Radiology.1946;47:487-91.

2. Miller DW. A review of proton beam radiation therapy. Med Phys. 1995;22:1943-54.

3. Hill-Kayser CE, Both S, Tochner Z. Proton Therapy: Ever Shifting Sands and the Opportunities and Obligations within. Front Oncol. 2011;1:24.

4. Fontenot JD, Lee AK, Newhauser WD. Risk of secondary malignant neoplasms from proton therapy and intensity-modulated x-ray therapy for early-stage prostate cancer. Int J Radiat OncolBiol Phys. 2009;74:616-22.

5. Levin WP, Kooy H, Loeffler JS, DeLaney TF.Proton beam therapy.Br J Cancer. 2005;93:849-54.

6. Newhauser WD, Giebeler A, Zhu R, et al. Uncertainty in dose per monitor unit estimates for passively scattered proton therapy: The role of compensator and patient scatter in prostate cases. Jour Proton Ther. 2015;1:116.

7. Rana S, Pokharel S, Zheng Y, et al. Treatment planning study comparing proton therapy, RapidArc and IMRT for a synchronous bilateral lung cancer case. Int J Cancer Ther Oncol. 2014; 2:020216.

8. Slater JD. Clinical applications of proton radiation treatment at Loma Linda University: review of a fifteen-year experience. Technol Cancer Res Treat. 2006;5:81-9.

9. Perez-Andujar A, Newhauser WD, Deluca PM. Neutron Production from beam modifying devices in a modern double scattering proton therapy beam delivery system. Phys Med Biol. 2009; 54:993-1008.

10. Zheng Y, Newhauser W, Klein E, Low D. Monte Carlo simulation of the neutron spectral fluence and dose equivalent for use in shielding a proton therapy vault.Phys Med Biol. 2009; 54:6943-57.

11. Agosteo S, Birattari C, Caravaggio M, et al. Secondary neutron and photon dose in proton therapy. RadiotherOncol.1998;48:293-305.

12. Binns PJ, Hough JH. Secondary dose exposures during 200 mev proton therapy. Radiat Prot Dosimetry. 1997;70:441-4.

13. Polf JC, Newhauser WD. Calculations of neutron dose equivalent exposures from range-modulated proton therapy beams. Phys Med Biol. 2005;50:3859-73.

14. Yan X, Titt U, Koehler AM, Newhauser WD. Measurement of neutron dose equivalent to proton therapy patients outside of the proton radiation field. NuclInstrum Methods Phys Res. 2002;476:429-34.

15. Schneider U, Agosteo S, Pedroni E, Besserer J. Secondary neutron dose during proton therapy using spot scanning. Int J Radiat Oncol Biol Phys. 2002;53:244-51.

16. Murray L, Henry A, Hoskin P, et al. Second primary cancers after radiation for prostate cancer: a review of data from planning studies. Radiat Oncol. 2013;8:172.

17. Brenner DJ, Hall EJ. Secondary neutrons in clinical proton radiotherapy: a charged issue. Radiother Oncol. 2008;86:165-170.

18. Stefano A, Allison J, AmakoK, et al. Geant4 - A simulation toolkit. Nucl Instrum Methods Phys Res. 2003; 506:250-303.

19. Allison J, Amako K, Araujo H, et al. Geant4 developments and applications. IEEE Trans Nucl Sci. 2006; 53:270-8.

20. Tesfamicael BY, Avery S, Gueye P, et al. Scintillating fiber based in-vivo dose monitoring system to the rectum in proton therapy of prostate cancer: A Geant4 Monte Carlo simulation. Int J Cancer Ther Oncol. 2014; 2:02024. 
21. Cirrone GAP, Cuttone G, Di Rosa F, et al. Validation of geant4 physics models for the simulation of the proton Bragg Peak. Proc. IEEE Nucl Sci Simp. 2006;6:788-92.

22. Cirrone GAP, Cuttone G, Di Rosa F, et al. The geant4 toolkit capability in the hadron therapy field: simulation of a transport beam line. Nucl Phys B - Proc Suppl. 2006;150:54-7.

23. Cirrone GAP, Cuttone G, Guatelli S, et al. Implementation of a new Monte Carlo-GEANT4 simulation tool for the development of a proton therapy beam line and verification of the related dose distributions. IEEE Trans Nucl Sci. 2005;52:262-5.
24. Cirrone GAP, Cuttone G,MazzagliaSE, et al. Hadrontherapy: a geant 4 based tool for proton/ion therapy studies. IEEE Trans Nucl Sci.2011;2:207-12.

25. Cirrone GAP, Cuttone G, Di Rosa F, et al. Monte Carlo based implementation of an energy modulation system for proton therapy. IEEE Nucl Sci Simp. 2004;4:2133-7.

26. Available from https://root.cern.ch/drupal/[Accessed on $1 / 21 / 2015]$

27. Wroe A, Rosenfeld A, Schulte R. Out-of-field dose equivalents delivered by proton therapy of prostate cancer. Med Phys.2007;34:3449-56. 